\title{
Proteomics of triple negative breast cancers in Asian population- the need for further studies
}

\begin{abstract}
Abbreviations: TNBC, triple negative breast cancers; ER, estrogen receptor; PR, progesterone receptor; BLBC, basal like breast cancer; TPI, triose phosphate isomerase; PGK, phosphoglycerate kinase; FASN, fatty acid synthase; HSP, heat shock protein; AKR, aldo-keto reductase; GLO, glyoxalase; ENO, enolase
\end{abstract}

\section{Introduction}

Reports from across Asia emphasize that breast cancer is becoming very common. It is projected that more than $70 \%$ of breast cancers will be from so-called 'developing countries' by 2020 , of which a very high proportion will be from Asia. ${ }^{1}$ Another important point is that there is genetic diversity between Western and Oriental population. There is a great need to develop new markers to identify breast cancer at an early stage. There is also a need for newer chemotherapeutic agents, which are more effective and less toxic. Cancers which are negative for ER, PR and HER2 are known as triple negative breast cancers (TNBC). They are very resistant to conventional treatment protocols and hence their management is one of the greatest challenges in contemporary clinical practice. Our own preliminary studies have identified 83 cases that did not express the 3 markers, ER, PR and HER2, i.e. triple negative breast cancer (TNBC) out of a total of 358 cases, $23.2 \%{ }^{2}$

One of the most aggressive types of sporadic breast cancers is those which are over-expressing the gene, HER-2/neu. It has been identified that $\sim 25 \%$ of breast cancers are over-expressing the HER-2/ neu gene. Cancers which over-express HER-2/neu genes are highly resistant to conventional therapies and also very aggressive in their biological characteristics, including local and distant metastasis. The combination of the 3 markers, estrogen receptor (ER), progesterone receptor (PR) and HER-2/neu is very commonly used for assessing treatment plan and prognosis. Management of triple negative breast cancers continues to be a challenging problem. Molecular phenotype of breast cancer is done on the basis of 5 markers, ER, PR, HER-2, CK 5/6 and EGFR. ER positive and/PR positive and HER2 negative are classified as luminal A, ER positive and/or PR positive and HER2 positive are luminal B cancers, ER and PR negative and HER2 positive are considered as HER2 type. Cancers which are negative for ER, PR and HER2 are known as triple negative breast cancers (TNBC). ER, PR and HER2 negative and positive for CK 5/6 and / or EGFR are categorized as basal-like cancers (BLBC). Those cancers negative for all 5 markers are considered as "unclassified" or "null". ${ }^{3}$

TNBC shares morphological and genetic abnormalities with basal like breast cancer (BLBC), but they are not the same entity. Breast cancers which carry BRCA1 mutations also very often come under TNBC/BLBC. They frequently occur in younger women and are aggressive and metastatic. They relapse more often and have a worse prognosis. Approximately $10-24 \%$ of breast cancers are TNBC. About $70 \%$ of TNBC are BLBC. TNBC and BLBC share many common characteristics like grade 3 status, ductal carcinomas with high mitotic count, high apoptotic rate, geographic or central necrosis and/ or fibrosis, a pushing border of invasion and stromal lymphocytic response. Hormone negativity, high grade, medullary-like histology,

\author{
Volume 5 Issue 5 - 2017 \\ Vaidyanathan $\mathrm{K}$ \\ Department of Biochemistry, Pushpagiri Institute of Medical \\ Science \& Research Center, India
}

\begin{abstract}
Correspondence: Vaidyanathan K, Professor \& Head, Department of Biochemistry, Pushpagiri Institute of Medical Science \& Research Center, Tiruvalla, Kerala, India,
\end{abstract} Email drkannan@pushpagiri.in

Received: February 13, 2017| Published: May 30, 2017

lymphocytic infiltration, TP53 mutations, EGFR expression, HER-2 negativity, characteristic copy number alterations and X-chromosome inactivation are termed as "BRCAness" because of their similarity to BRCA1 mutation carriers. Thus, there is considerable overlap between the three entitites, namely, BLBC, TNBC and BRCA1 mutation carriers. ${ }^{4}$

Protein profiling as well as phosphoproteomics of the triple negative cases would identify novel molecules that are involved in the development/progress of the cancer. Over expression of Cyclin E1 ${ }^{5}$ metastasis-related proteins ${ }^{6}$ as well as glycolytic enzymes, cytokeratins, and structural proteins ${ }^{7}$ were seen in triple negative breast cancers. Gromov et al., ${ }^{8}$ identified 46 proteins that were upregulated in triple negative tumors. Zhang et al., ${ }^{9}$ have studied proteomic signature in 3 breast cancer cell lines of inflammatory breast cancer (SKBR3, SUM149 and SUM190). In a study by Sohn et al., ${ }^{10}$ AKT, IGFBP2, LKB1, S6 and Stathmin were predictors of recurrence-free survival in triple negative breast cancer patients undergoing standard neoadjuvant chemotherapy. Gamez-Pozo et al., ${ }^{11}$ have studied proteome of TNBC and identified and quantified more than 1000 protein groups, including CD44 and PARP1. They have identified signaling pathways which they report is related to TNBC genesis and development. Zhang et al., ${ }^{12}$ did integrative pathway assisted proteomic analysis and report clustering of breast cancer subtypes. Luminal A and B sub-types resembled each other which were different from basal group (basal and HER-2 types). Their results suggest the importance of molecular classification and warrants further proteomic studies based on molecular sub-types.

Proteins of cytoskeletal organization, calcium binding, and stress response were identified in ER positive/HER2 negative breast cancers by Korwar et al. ${ }^{13}$ Interactions of annexin A5, actin, S100 A10, glyceraldehyde 3 phosphate dehydrogenase, superoxide dismutase 1 , apolipoprotein, fibrinogen, and heat shock proteins were also found. Pavlou et al., ${ }^{14}$ performed MS-based secretome analysis of eight breast cancer cell lines, corresponding to the three main breast cancer subtypes and more than 5200 non-redundant proteins were identified with 23 , four, and four proteins identified uniquely in basal, HER2-neu-amplified, and luminal breast cancer cells, respectively. Cabezon et al., ${ }^{15}$ performed proteomic studies on TNBC and identified Mage-A4 protein as a potential drug target. 
Perez-Rivas et al., ${ }^{16}$ report distinct changes in proteomic profile following breast cancer surgery. Terp et al., ${ }^{17}$ studied key proteins associated with the metastatic potential of breast cancer cells and identified LRRC59, CD59 and CSPG4. Proteins involved in cell growth stimulation, anti-apoptosis mechanisms and cancerogenesis were more strongly expressed in T47D than in MCF7, while, proteins implicated in transcription repression and apoptosis regulation, were more strongly expressed in MCF7 as compared to T47D in a study by Aka et al. ${ }^{18}$

Greenwood et al., ${ }^{19}$ suggest that triple-negative breast tumors can be segregated into 2 phenotypes based on their genome-wide protein abundance profiles. One of the groups, Stat1/CD74 positive triplenegative tumors, was more aggressive compared to the other group. He et al., ${ }^{20}$ identified that twenty proteins were found to correctly classify all HER 2 positive and 7 of the 11 TNBC tumors. Among them, galectin-3-binding protein and ALDH1A1 were found preferentially elevated in TNBC, whereas CK19, transferrin, transketolase, and thymosin $\beta 4$ and $\beta 10$ were elevated in HER2-positive cancers.

Gonzalez-Angulo et al., ${ }^{21}$ identified a 10 -protein biomarker panel that classifies breast cancer into prognostic groups that may have potential utility in the management of patients who receive anthracycline-taxane-based NST. These proteins are - ER function (ER, PR, Bcl2, GATA3, EIG121), tyrosine kinase receptor function (EGFR, HER2, HER2p1248), and cell proliferation (CCNB1, CCNE1). Chen et al., ${ }^{22}$ identified 23 proteins over expressed in HER2 positive breast cancers, including creatine kinase, retinol-binding protein 1 , thymosin 4 and tumor protein D52. Schulz et al., ${ }^{23}$ compared the protein expression pattern of triple-negative breast carcinomas versus those being positive for HER2 and negative for the hormone receptors (HER2+, ER-, PR-) by 2-D DIGE and mass spectrometry. They obtained differential expression patterns for several glycolytic enzymes (MDH2, PGK1, TKT, Aldolase1), cytokeratins (CK7, 8, 9, 14, 17, 19), further structure proteins (vimentin, fibronectin, L-plastin), for NME1-NME2, lactoferrin, and members of the Annexin family. They suggest that the identified marker proteins may advance a more detailed characterization of triple-negative breast cancers and may contribute to the development of better treatment strategies.

Zhang et al., ${ }^{24}$ identified nine proteins involved in glycolysis (triose-phosphate isomerase (TPI), phosphoglycerate kinase 1 (PGK1), and enolase 1 (ENO1)), lipid synthesis (fatty acid synthase (FASN)), stress-mediated chaperonage (heat shock protein 27 (Hsp27)), and antioxidant and detoxification pathways (haptoglobin, aldo-keto reductase (AKR), glyoxalase I (GLO), and prolyl-4hydrolase beta-isoform (P4HB)) were found to be up-regulated in HER-2/neu-positive breast tumors. They suggest that HER-2/neu signaling may result, directly or indirectly, in enhanced activation of various metabolic, stress-responsive, antioxidative, and detoxification processes within the breast tumor microenvironment.

Costa et al. ${ }^{25}$ studied non-tumoral breast tissue. Forty-four different proteins from 70 spots were identified and classified according to their biological function. Cytoskeleton and associated proteins represented the largest class $(30 \%)$ followed by the proteins with binding function $(27 \%)$. Several of the proteins were also seen in breast tumors, such as vimentin, endoplasmin, small heat shock beta- 6 , disulfide isomerase and some cell growth, and proliferation regulators. This suggests the importance of including data on the characterization of non-tumoral breast and to studies on differential expression in cancer tissue. Many hydrophobic proteins were found to be shared between TNBC and $\mathrm{ER}+\mathrm{PR}+$ Her2-breast cancers (non-TNBC). Unique proteins were also found to be associated with either TNBC or ER+PR+Her2-breast cancers. The pool of the unique proteins may include disease related biomarkers, and may potentially be used as therapy targets. ${ }^{26}$ Labelfree quantitative proteome analysis revealed that 236 proteins were differentially expressed in ER $\alpha+$ and ER $\alpha$-breast tumors. By Gene Ontology molecular function, dehydrogenase, reductase, cytoskeletal proteins, extracellular matrix, hydrolase, and lyase categories were significantly enriched in ER $\alpha+$, whereas selected calcium-binding protein, membrane traffic protein, and cytoskeletal protein were enriched in ER $\alpha$-breast tumors. ${ }^{27}$ It would be interesting to follow future studies on triple negative breast cancer proteomics from the Asian population and the molecular signature of these patients.

\section{Acknowledgements}

None.

\section{Conflict of interest}

The author declares no conflict of interest.

\section{References}

1. Flockhart D. Germline pharmacogenomics as a tool to individualize therapy in breast cancer. In: Innocenti F, Liu G, editors. Cancer Therapy: Latest thinking in efficacy and toxicity. UK: The Biomedical \& Life Sciences Collection, Henry Stewart Talks Ltd; 2009.

2. Vaidyanathan K, Kumar P, Reddy CO, et al. ErbB-2 expression and its association with other biological parameters of breast cancer among Indian women. Indian J Cancer. 2010;47(1):8-15.

3. Sorlie T, Tibshirani R, Parker J, et al. Repeated observation of breast tumor subtypes in independent gene expression data sets. Proc Natl Acad Sci USA. 2003;100(14):8418-8423.

4. Carey L, Winer E, Viale G, et al. Triple-negative breast cancer: disease entity or title of convenience? Nat Rev Clin Oncol. 2010;7(12):683-692.

5. Agarwal R, Gonzalez-Angulo AM, Myhre S, et al. Integrative Analysis of Cyclin Protein Levels Identifies Cyclin B1 as a Classifier and Predictor of Outcomes in Breast Cancer. Clin Cancer Res. 2009;15(11):3654-3662.

6. Sun B, Zhang S, Zhang D, et al. Identification of Metastasis-Related Proteins and their Clinical Relevance to Triple-Negative Human Breast Cancer. Clin Cancer Res. 2008;14(21):7050-7059.

7. Schulz DM, Böllner C, Thomas G, et al. Identification of differentially expressed proteins in triple-negative breast carcinomas using DIGE and mass spectrometry. J Proteome Res. 2009;8(7):3430-3438.

8. Gromov P, Gromova I, Bunkenborg J, et al. Up-regulated proteins in the fluid bathing the tumour cell microenvironment as potential serological markers for early detection of cancer of the breast. Mol Oncol. 2010;4(1):65-89.

9. Zhang EY, Cristofanilli M, Robertson F, et al. Genome wide proteomics of ERBB2 and EGFR and other oncogenic pathways in inflammatory breast cancer. J Proteome Res. 2013;12(6):2805-2817.

10. Sohn J, Do KA, Liu S, et al. Functional proteomics characterization of residual triple-negative breast cancer after standard neoadjuvant chemotherapy. Ann Oncol. 2013;24(10):2522-2526.

11. Gámez-Pozo A, Ferrer NI, Ciruelos E, et al. Shotgun proteomics of archival triple-negative breast cancer samples. Proteomics Clin Appl. $2013 ; 7(3-4): 283-291$.

12. Zhang F, Chen JY. Breast cancer subtyping from plasma proteins. $B M C$ Med Genomics. 2013;6(Suppl 1):S6.

13. Korwar AM, Bhonsle HS, Ghole VS, et al. Proteomic profiling and interactome analysis of ER-positive/HER2/neu negative invasive ductal carcinoma of the breast: towards proteomics biomarkers. OMICS. 2013;17(1):27-40 
14. Pavlou MP, Dimitromanolakis A, Diamandis EP. Coupling proteomics and transcriptomics in the quest of subtype-specific proteins in breast cancer. Proteomics. 2013;13(7):1083-1095.

15. Cabezón T, Gromova I, Gromov P, et al. Proteomic profiling of triplenegative breast carcinomas in combination with a three-tier orthogonal technology approach identifies Mage-A4 as potential therapeutic target in estrogen receptor negative breast cancer. Mol Cell Proteomics. 2013;12(2):381-394.

16. Perez-Rivas LG, Jerez JM, Fernandez-De Sousa CE, et al. Serum protein levels following surgery in breast cancer patients: a protein microarray approach. Int J Oncol. 2012;41(6):2200-2206.

17. Terp MG, Lund RR, Jensen ON, et al. Identification of markers associated with highly aggressive metastatic phenotypes using quantitative comparative proteomics. Cancer Genomics Proteomics. 2012;9(5):265273.

18. Aka JA, Lin SX. Comparison of functional proteomic analyses of human breast cancer cell lines T47D and MCF7. PLoS One. 2012;7(2):e31532.

19. Greenwood C, Metodieva G, Al-Janabi K, et al. Stat1 and CD74 over expression is co-dependent and linked to increased invasion and lymph node metastasis in triple-negative breast cancer. J Proteomics. 2012;75(10):3031-3040.

20. He J, Whelan SA, Lu M, et al. Proteomic-based biosignatures in breast cancer classification and prediction of therapeutic response. Int $J$ Proteomics. 2011;2011:896476.
21. Gonzalez-Angulo AM, Hennessy BT, Meric-Bernstam F, et al. Functional proteomics can define prognosis and predict pathologic complete response in patients with breast cancer. Clin Proteomics. 2011;8(1):11.

22. Chen H, Pimienta G, Gu Y, et al. Proteomic characterization of Her2/ neu-over expressing breast cancer cells. Proteomics. 2010;10(21):38003810 .

23. Schulz DM, Böllner C, Thomas G, et al. Identification of differentially expressed proteins in triple-negative breast carcinomas using DIGE and mass spectrometry. J Proteome Res. 2009;8(7):3430-3438.

24. Zhang D, Tai LK, Wong LL, et al. Proteomic study reveals that proteins involved in metabolic and detoxification pathways are highly expressed in HER-2/neu-positive breast cancer. Mol Cell Proteomics. 2005;4(11):1686-1696.

25. Costa GG, Kaviski R, Souza LER, et al. Proteomic analysis of nontumoral breast tissue. Genet Mol Res. 2011;10(4):2430-2442.

26. Lu M, Whelan SA, He J, et al. Hydrophobic Proteome Analysis of Triple Negative and Hormone-Receptor-Positive-Her2-Negative Breast Cancer by Mass Spectrometer. Clin Proteomics. 2010;6(3):93-103.

27. Rezaul K, Thumar JK, Lundgren DH, et al. Differential Protein Expression Profiles in Estrogen Receptor-Positive and Negative Breast Cancer Tissues Using Label-Free Quantitative Proteomics. Genes Cancer. 2010;1(3):251-271. 